\title{
Reporting of outcomes in arthritis trials measured on ordinal and interval scales is inadequate in relation to meta-analysis
}

P C Gøtzsche

\begin{abstract}
Objectives-To study whether the reporting of clinical outcomes in arthritis trials measured on ordinal and interval scales is adequate in relation to meta-analysis.

Methods-Systematic review of randomised trials of non-steroidal antiinflammatory drugs in patients with rheumatoid arthritis. Optimal reporting was defined as data in the original ordered categories for global evaluation and pain, and as mean and SD for number of tender joints and grip strength, and if a visual analogue scale had been used to measure pain.

pain.

Results-A total of 144 trials were included. The median sample size was 60 patients. The quality of the reporting increased over time for three of the four variables. Global evaluation was optimally reported in 52 of the 127 trials $(41 \%)$ in which it was recorded. Pain was optimally of tender joints in 41 of 123 trials $(33 \%)$, and grip strength in 34 of 124 trials $(27 \%)$. and grip strength in 34 of 124 trials (27\%). Even if rather broad criteria are adopted, in a potentially useful way for a metaanalysis.

Conclusions-Arthritis trials have been reported inadequately in relation to metaanalysis. As most trials are underpowanalysis. As most trials are underpowered, meta-analysis is indispensable and the deficit therefore needs urgent improvement. Investigators should specify a priori what constitutes an important treatment effect and report numbers of patients improved.

(Ann Rheum Dis 2001;60:349-352)
\end{abstract}

It is an important challenge to the medical community how best to extract and combine the information that is contained in reports of randomised clinical trials. The total number of trials has proved to be much larger than was expected just a few years ago. In 1994, only

The Nordic Cochrane Centre, Rigshospitalet, Department 7112 DK-2100 Copenhagen DK, Denmar: Ø, Denmark

Correspondence to: Dr Gøtzsche p.c.gotzsche@cochrane.dk Accepted 12 September
19000 citations on Medline were identifiable

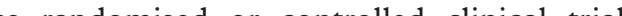
as randomised or controlle Largely because of the systematic electronic and hand searches performed by members of the Cochrane Collaboration, this number has increased to more than 180000 , and the most comprehensive source of citations to trials, The Cochrane Controlled Trials Register, currently contains more than 250000 references. ${ }^{2}$

As most trials are too small to answer the question they address, ${ }^{3}{ }^{4}$ it is usually necessary to aggregate the results in meta-analyses. In most trials, the primary outcomes have been measured on an ordinal scale with graded classes - for example, severity of pain - or on an interval scale-for example, blood pressure. ${ }^{4}$ This presents a challenge for metaanalysts, because the reporting of such data is ften poor ${ }^{5}$ It may therefore not be possible to require that numbers of patients in ordered or binary categories or means and standard deviations are reported.

To make the most of the available information for clinical decision making and research planning, it may be necessary to use more crude, less starting it work, it is useful to know what the main problems are. I studied the reporting of data measured on ordinal and interval scales in systematic review of trials of non-steroidal antiinflammatory drugs (NSAIDs) in patients with heumatoid arthritis.

\section{Methods}

EARCH STRATEGY

collected reports on group comparative randomised double blind trials that compared two of the 22 NSAIDs currently marketed in Denmark. The trials were of tablets or capsules, given in repeated doses to patients with rheumatoid arthritis, and published before 1998. I excluded trials on several diseases oid arthritis separately, and trials published as abstracts. A Medline search was carried out in August 1998. "Arthritis, rheumatoid" (exploded) was combined with (review in publication type or comparative-study in check tag or "dose-response relationship, drug" (exploded)) and with one or more of the drugs as exploded. I scanned the reference lists of the collected articles. In the case of repetitive publications, the most informative was chosen. Th searches identified 156 potentially eligible trials. I excluded five trials in Japanese, one in Russian and one in Serbo-Croat because of the anguage. Another five trials were excluded as via the niversity library. The final sample consisted of 44 trials in which one or more of the four selected outcomes mentioned below had been measured (references are available on request)

DATA EXTRACTION

Data were extracted on year of publication, number of patients, and on the following clinical outcomes: global evaluation of the effect, 

Table 1 Proportion of trials with optimal and usable reporting for meta-analysis (see
definitions in text). Test for positive trend

\begin{tabular}{|c|c|c|c|c|c|}
\hline & $1966-77$ & $1978-82$ & $1983-87$ & $1988-97$ & $\begin{array}{l}\text { Trend } \\
\text { ( } p \text { value) }\end{array}$ \\
\hline \multicolumn{6}{|l|}{ Global evaluation } \\
\hline No of trials & 35 & 31 & 33 & 28 & \\
\hline Optimal reporting (\%) & 43 & 52 & 33 & 36 & 0.32 \\
\hline \multirow{2}{*}{\multicolumn{6}{|c|}{ Pain }} \\
\hline & & & & & \\
\hline No of trials & 16 & 27 & 25 & 30 & \\
\hline Optimal reporting (\%) & 13 & 15 & 28 & 47 & 0.003 \\
\hline Usable reporting (\%) & 50 & 30 & 48 & 63 & 0.09 \\
\hline \multicolumn{6}{|l|}{ No of tender joints } \\
\hline No of trials & 31 & 30 & 31 & 31 & \\
\hline Optimal reporting (\%) & 19 & 20 & 39 & 55 & 0.001 \\
\hline Usable reporting (\%) & 29 & 27 & 45 & 58 & 0.007 \\
\hline \multicolumn{6}{|l|}{ Grip strength } \\
\hline No of trials & 40 & 28 & 30 & 25 & \\
\hline Optimal reporting (\%) & 18 & 25 & 33 & 40 & 0.03 \\
\hline
\end{tabular}

pain, number of tender joints, and grip strength. These four outcomes are commonly used in NSAID trials ${ }^{5}$ and they are measured on an ordinal scale or on an interval scale.

I noted whether the reported data could be used in a standard meta-analysis and whether the reporting improved over time. To allow time trend analyses, I separated the trials into four groups of approximately equal size.

To be optimally reported, data on global evaluation should be available in the original ordered categories. Thus, if a five point scale had been used but only numbers of patients who had improved were reported, this outcome was not optimally reported. Grip strength should be reported as mean after treatment or mean change, and standard deviations should be available for absolute values or for changes, as appropriate, or should be estimable-for example, from exact $\mathrm{p}$ or $\mathrm{t}$ values. The same applied to number of tender joints. Pain data should be available in the original ordered categories; if a visual analogue scale had been used, mean and SD were also accepted.

used, mean and SD were also accepted.
In a secondary analysis, I noted whether the data were potentially usable for a meta-analysis in a rather broad sense. I only required that the data were reported in two or more ordered categories, or as mean or mean change with corresponding SD.
The Jonckheere-Terpstra test was used for time trend analysis of sample size (MEDSTAT program); proportions were analysed with a $\chi$ test for trend (BMDP program).

\section{Results}

The 144 trials included comprised a total of 16659 patients. The median sample size was 60 patients; it increased over time $(p=0.01)$ and was 110 in the most recent time period, 1988-1997.

Table 1 shows the usefulness of the reported outcomes for meta-analysis. Global evaluation was recorded in 127 trials. It was optimally reported in 52 trials $(41 \%)$ and usefully reported in 88 trials $(69 \%)$. There was a nonsignificant trend towards poorer reporting in the most recent years $(p=0.32$ for optimal reporting and $\mathrm{p}=0.15$ for useful reporting)

Pain was recorded in 98 trials. It was optimally reported in 27 trials $(28 \%)$ and usefully reported in 47 trials $(48 \%)$. There was a significant trend towards better reporting over time ( $\mathrm{p}=0.003$ and $\mathrm{p}=0.09$ respectively).

Number of tender joints, or closely similar measures such as Ritchie's joint count index, was recorded in 123 trials. It was optimally reported in 41 trials $(33 \%)$ and usefully reported in 49 trials $(40 \%)$. There was a was snificant trend towards better reporting over

Grip strength was recorded in 124 trials. It
( $=0.001$ and $p=0.007$ respectively). was optimally reported in 34 trials $(27 \%)$ and usefully reported in 41 trials (33\%). There was a significant trend towards better reporting over time ( $\mathrm{p}=0.03$ and $\mathrm{p}=0.07$ respectively)

Table 2 shows the mode of reporting in . ack of standard deviations of the measurements. Confidence intervals, standard errors, exact $\mathrm{p}$ or $\mathrm{t}$ values, or other relevant test statistics that would have allowed the calculation of standard deviations, were also missing. Twenty out of 127 reports $(16 \%)$ provided no data at all on 127 reports (16\%) provided no data at out of $123(18 \%)$ reports on pain.

Table 2 Method of data presentation in 144 reports of trials comparing two non-steroidal anti-inflammatory drugs

\begin{tabular}{|c|c|c|c|c|}
\hline Method of data presentation & $\begin{array}{l}\text { Global } \\
\text { evaluation }\end{array}$ & Pain & $\begin{array}{l}\text { Number of } \\
\text { tender joints }\end{array}$ & $\begin{array}{l}\text { Grip } \\
\text { strength }\end{array}$ \\
\hline \multicolumn{5}{|l|}{ Usable reporting } \\
\hline Numbers of patients in original ordered categories & 52 & 7 & & \\
\hline Numbers in reduced ordered categories, at least 3 & 12 & 5 & 4 & 3 \\
\hline Numbers in only 2 (reduced) ordered categories & 14 & 5 & 4 & \\
\hline Mean and SD, before and after treatment & 4 & 26 & 35 & 29 \\
\hline Mean and SD before, mean after treatment & 1 & 1 & 2 & \\
\hline Mean and SD after treatment & 4 & 1 & 1 & \\
\hline Mean change and SD for change & 1 & 2 & 3 & 5 \\
\hline \multicolumn{5}{|l|}{ Unusable reporting } \\
\hline Mean before and after treatment, no SD & 8 & 26 & 46 & 44 \\
\hline Mean after treatment, no SD & 6 & & 1 & \\
\hline Mean change, no SD & 5 & 6 & 9 & 9 \\
\hline Percentage change, no SD & & 1 & 4 & 2 \\
\hline Median and $\mathrm{IQ}^{\star}$ range, before and after treatment & & & 1 & 2 \\
\hline Median change and IQ range & & & & 1 \\
\hline Geometric mean percentage change, no SD & & 3 & 4 & 1 \\
\hline Median change, no percentiles or SD & & 2 & 2 & 2 \\
\hline Median before and after treatment, no centiles & & & 1 & \\
\hline No data shown & 20 & 13 & 6 & 21 \\
\hline Total number of trials reporting the variable & 127 & 98 & 123 & 123 \\
\hline
\end{tabular}

${ }^{\star} \mathrm{IQ}=$ Interquartile. 
Discussion

The reporting quality improved over time, but even in the most recent time period, and even if rather broad criteria were adopted, only about half of the data were reported in a way that made them potentially useful for a metaanalysis. This deficit in reporting was similar in small and large trials. For pain, for example, $48 \%$ of the trial reports were useful, and if the unit of analysis was patients, this proportion was very similar, $49 \%$.

It was surprising that not a single confidence interval was presented in any of the 34 most recent trial reports, as all the reviewed trials aimed to compare the effect and tolerability of two similar drugs. Such trials are pragmatic, as the questions they address are directly relevant for clinical decision making. The $95 \%$ confidence interval allows the reader to judge whether a clinically relevant difference can be ruled out with reasonable certainty. Without confidence intervals, the non-statistically minded clinician may be misled into believing curious claims such as the new drug is "at least as effective" ${ }^{5}$ as the standard drug. As such claims are often accompanied by doubtful or biased statements about less toxicity, ${ }^{5}$ these trials seem primarily to have served marketing purposes.

Most of the trials were considerably underpowered, even if the sample size estimation is based on the patient's global evaluation of the effect, which is the most sensitive outcome. ${ }^{6}$ If, in accordance with the examples above, one wishes not to overlook the possibility that a new drug may be only half as effective as the standard drug, the necessary sample size is 290 patients for a power of $80 \% .{ }^{67}$ As NSAIDs are patients for a power of $80 \%$.
effective drugs, ${ }^{6}$ a decrease in effect of $25 \%$ could also be clinically relevant to detect, in which case 1160 patients would be needed.

When sample sizes are too small, it is particularly important that the results are reported in a way that facilitates meta-analysis. Even though small trials tend to exaggerate the treatment effect, ${ }^{8}$ they can sometimes be useful for meta-analysis if the aggregated result is interpreted cautiously and the likely magnitude and direction of bias is taken into account. For example, it has been shown in meta-analyses of small trials with ordinal and interval scale outcomes that low dose prednisolone is highly effective in rheumatoid arthritis ${ }^{9}$ and, conversely, that chemical and physical measures to decrease exposure to house dust mites are ineffective in patients with asthma. ${ }^{10}$

For a symptomatic treatment such as an NSAID, the global evaluation of the effect is an important outcome because it allows the patient to express the various benefits and adverse effects of the drug in a simple measure. As this outcome is almost always recorded on a As this outcome is almost always recorded on a
four or five point ranking scale, it was surprisfour or five point ranking scale, it was surpris-
ing that the data were reported in the original ordered categories in only $52(41 \%)$ of the trials (table 2). In 26 trials (20\%), the data were reduced to a scale with only two or three categories before reporting, which creates a risk of bias, as the egories and cut offs between them may not be independent of which drug will be favoured. It is also problematic for the validity of a meta-analysis that no data were shown for global evaluation in as many as $16 \%$ of the trials, as one would not expect such omissions to ccur when the result favours the new drug over the standard. ${ }^{5}$

These problems could be minimised if research ethics committees ensured that raw trial data became publicly available after a period of time that allows the investigators to publish their results. By making unpublished ided. As long as this has not happened d, howver, it remains an important challenge fo meta-analysts to determine how to take stock of all the imperfectly reported results. For example, trials could be weighted by their sample size rather than by their inverse variance, ${ }^{11}$ and missing standard deviations could be estimated from similar trials. It is not the purpose of this article, however, to explore these possibilities.

Previous surveys of statistical reporting of andomised trials seem not to have addressed the objective of this survey-that is, whether the reporting allows the data to be used for meta-analysis. It is a limitation of the survey that only one drug category was included. On generalised. Most clinical trials are drug trials supported by industry and many of the largest companies have developed and marketed NSAIDs. A similar reporting pattern would therefore be expected for many other trials sponsored by drug companies. Furthermore, the types of outcomes I have reviewed are very in fact, most trials in health care have a primary outcome that has been measured on an ordinal or an interval scale.

It can therefore be assumed that a large body of trials of healthcare interventions have been reported inadequately in relation to metaanalysis. This deficit needs urgent improvement. It would also be helpful if investigators always specified a priori what constituted an important treatment effect and reported numbers of patients who improved. This would lead to a minor decrease in statistical power which seems acceptable, in particular as there is an importan gain in clinical relevance. It is difficult to know whether an improvement of $1.5 \mathrm{~cm}$ on a $10 \mathrm{~cm}$ pain scale or some points on a composite score pereas it is obviously relevant if a severe pain or depression has gone.

The study was funded by the Danish Medical Research Coun-

1 Lefebvre C, Clarke M. Identifying randomised trials for sys-

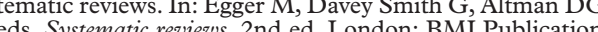
The Cochrane Library. Oxford: Update Software. Updated quarterly Issue 2, 2000. importance of beta, the type II error and sample size in the design and interpretation of the randomized control tria: 4 Mulward S, Gøtzsche PC. Sample size of randomized
double-blind trials 1976-1991. Dan Med Bull 1996;43: double

(1976-1991. Dan Med Bull 1096:43: 5 Gøtzsche PC. Methodology and overt and hidden bias flammatory drugs in rheumatoid arthritis. Control Clin 


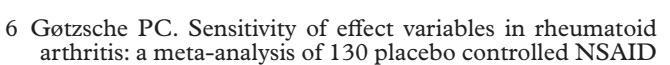
arthritis: a meta-analysis of 130 placebo controlled NSAID
trials. J Clin Epidemiol 1990;43:1313-18. 7 Pocock SJ. Clinical trials: a practical approach. Chichester: 8 John Wiley \& Sons, 1983. analysis detected by a

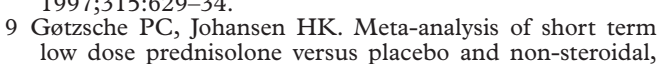

anti-inflammatory drugs in rheumatoid arthritis. BMJ
1998:316:811-18. Also published in The Cochrane Library, Issue 3, 2000. Oxford: Update Software.
Gøtzsche PC, Hammarquist C, Burr M. House dust mite control measures in the management of asthma: metaCochrise Library 11 Laird NM, Mosteller F. Some statistical methods fo combining experimental results. Int J Technol Assess 